NOTA

\title{
DIVERSIDADE DE AFÍDEOS NA CULTURA DO ALGODOEIRO NO MUNICÍPIO DE CAMPO VERDE (MT) ${ }^{(1)}$
}

\author{
MARCOS DONISETI MICHELOTTOO ${ }^{(2)}$; ANTONIO CARLOS BUSOLI ${ }^{(3)}$
}

\begin{abstract}
RESUMO
O objetivo deste trabalho foi verificar a diversidade de afídeos (Hemiptera: Aphididae) na cultura do algodoeiro (Gossypium hirsutum L.) no município de Campo Verde (MT). Os afídeos foram amostrados diretamente nas plantas e através de armadilhas tipo Moericke. As amostragens foram realizadas a cada dois dias, até 60 dias após a germinação das plantas. A espécie Aphis gossypii Glover prevaleceu nas amostragens realizadas sobre as plantas. Formas aladas, de ocorrência acidental na cultura, tais como Aphis spiraecola Patch e Rhopalosiphum padi (Linnaeus) também foram observadas. Com as armadilhas tipo Moericke foram capturados 2280 afídeos alados, pertencentes a 13 espécies: $R$. padi (52,6\% do total), A. spiraecola $(26,4 \%)$, A. gossypii (8,9\%), Rhopalosiphum maidis (Fitch) (5,3\%), Geopemphigus floccosus (Moreira) (3,1\%), Uroleucon ambrosiae (Thomas) (1,5\%), Rhopalosiphum rufiabdominalis (Sasaki) (1,3\%), Myzus persicae (Sulzer) $(0,4 \%)$, Sipha flava (Forbes) (0,3\%), Pentalonia nigronervosa Coquerel, Tetraneura nigriabdominalis (Sasaki), Lizerius melanocallis (Quednau) e Toxoptera citricidus (Kirkaldy) (0,1\% cada uma). Nas amostragens diretamente sobre as plantas foram observados ápteros e alados de A. gossypii e alados de A. spiraecola e $R$. padi. Nas armadilhas tipo Moericke, as principais espécies capturadas foram R. padi, A. spiraecola, A. gossypii e R. maidis.
\end{abstract}

Palavras-chave: Aphididae, amostragem, Gossypium, relação afídeo/hospedeiro, armadilha amarela.

\section{ABSTRACT \\ APHIDS DIVERSITY ON COTTON FIELD IN CAMPO VERDE, MATO GROSSO STATE}

This work was carried out in Campo Verde, Mato Grosso State, Brasil, and its objective was to determine the occurrence of aphid species (Hemiptera: Aphididae) on cotton field (Gossypium hirsutum L.). The aphids were sampled both directly on plants and with Moericke's traps. The samplings were accomplished every other day during 60 days. Aphis gossypii Glover prevailed on cotton plants. Winged forms accidental as Aphis spiraecola Patch and Rhopalosiphum padi (Linnaeus) also occurred. In the waterpan traps 2280 winged aphids were trapped, which are from 13 species: R. padi ( $52.6 \%$ of total), A. spiraecola (26.4\%), A. gossypii (8.9\%), Rhopalosiphum maidis (Fitch) (5.3\%), Geopemphigus floccosus (Moreira) (3.1\%),

( $\left.{ }^{1}\right)$ Recebido para publicação em 15 de abril de 2002 e aceito em 14 de fevereiro de 2003.

$\left(^{2}\right)$ Pós-graduando em Entomologia Agrícola, Faculdade de Ciências Agrárias e Veterinárias/UNESP, Via de Acesso Prof. Paulo Donato Castellane, s/no , 14884-900 Jaboticabal (SP). E-mail: michelot@fcav.unesp.br

$\left(^{3}\right)$ Departamento de Fitossanidade, Faculdade de Ciências Agrárias e Veterinárias/UNESP. E-mail: acbusoli@fcav.unesp.br 
Uroleucon ambrosiae (Thomas) (1.5\%), Rhopalosiphum rufiabdominalis (Sasaki) (1.3\%), Myzus persicae (Sulzer) $(0.4 \%)$, Sipha flava (Forbes) $(0.3 \%)$, Pentalonia nigronervosa Coquerel (0.1\%), Tetraneura nigriabdominalis (Sasaki) $(0.1 \%)$, Lizerius melanocallis (Quednau) $(0.1 \%)$ and Toxoptera citricidus (Kirkaldy) (0.1\%). In the direct samplings on cotton plants were present both wingless and winged forms of the $A$. gossypii and winged forms of the $A$. spiraecola and $R$. padi. In the Moericke's traps, the main captured species are $R$. padi, $A$. spiraecola, A. gossypii and R. maidis.

Key words: Aphididae, sampling, Gossypium, aphid/host relation, yellow trap.

\section{Introdução}

O algodoeiro, Gossypium hirsutum L., é uma planta atacada por diversos insetos, que podem reduzir a produtividade e a qualidade das sementes e fibras (SANTOS, 1999). Dentre esses, destacam-se os afídeos, pois além de sugarem a seiva, interferindo no desenvolvimento das plantas (CALCAGNOLO e SAUER, 1954; Godfrey et al., 2000), são eficientes vetores de vírus fitopatogênicos (Peña-Martinez, 1992; Costa et al., 1997).

De acordo com BlackMAn e EASTOP (1984), as espécies Aphis maidiradicis Forbes, Rhopalosiphum rufiabdominalis (Sasaki), Macrosiphum euphorbiae (Thomas), Aphis craccivora Koch, Aphis fabae Scopoli, Smynthurodes betae Westwood, Acyrtosiphon gossypii Mordvilko, Myzus persicae (Sulzer) e Aphis gossypii Glover, foram observadas colonizando a cultura do algodoeiro. Segundo Stoetzel et al. (1996), com exceção de Acyrtosiphon gossypii, as demais espécies que ocorrem em algodoeiros nos Estados Unidos são as mesmas citadas por BLACKMAN e EASTOP (1984).

As espécies Aphis gossypii, M. persicae, M. euphorbiae e Aphis medicaginis Koch são mencionadas como pragas na cultura do algodoeiro na Colômbia (OсноA, 1989). No Brasil, as espécies encontradas são principalmente $A$. gossypii e ocasionalmente $M$. persicae, sendo a primeira vetora dos vírus do vermelhão e do vírus do mosaico das nervuras forma Ribeirão Bonito (CiA, 1977; SANTOS, 1999).

Nos últimos anos, com a grande utilização de cultivares oriundas de materiais de outros países, as viroses se tornaram as principais doenças da cultura do algodoeiro, principalmente na região Centro-Oeste do Brasil (FreIRE, 1999). No ano agrícola de 1998/99, 75\% da área de algodoeiro no Estado de Mato Grosso se baseou nas cultivares CNPA ITA 90 e DELTAPINE ACALA 90, mesmo sendo consideradas altamente suscetíveis ao vírus do mosaico das nervuras forma Ribeirão Bonito (FREIRE, 1999; SANTOS 1999).

O objetivo do presente trabalho foi estudar a diversidade de afídeos no início de desenvolvimento da cultura do algodoeiro em Campo Verde (MT), através da visualização direta das plantas e armadilhas tipo Moericke.

\section{Material e Métodos}

Área de amostragem de afídeos

O trabalho foi realizado em uma área de aproximadamente $1.700 \mathrm{~m}^{2}$, na Fazenda Mourão, município de Campo Verde (MT), em janeiro e fevereiro de 2001. Foram utilizadas sementes da cultivar CNPA ITA 90 previamente tratadas com carbofuram (Furadam 350 TS, 2,0 L/100 kg de sementes) e carboxin-thiran (Vitavax-Thiram 200 SC, 0,5 L/100 kg de sementes).

Após a emergência das plântulas, realizaramse amostragens a cada dois dias, por um período de 60 dias (25 amostragens). VENDRAMIM e NAKANO (1981) consideraram o referido período como o mais crítico ao ataque dos afídeos na cultura do algodoeiro. Os seguintes métodos de amostragens foram utilizados:

a) Amostragem visual. Avaliaram-se 20 plantas ao acaso, de modo a abranger toda a área cultivada. Dessas plantas, os afídeos presentes foram retirados com o auxílio de um pincel e depositados em frascos de vidro de $10 \mathrm{~mL}$, contendo álcool $70 \%$, vedados com tampa de borracha e etiquetados com suas respectivas datas de coleta. Posteriormente, o material foi levado ao laboratório e observado em estereoscópio.

Os insetos foram separados de acordo com as suas características morfológicas e contados. Exemplares de cada morfo-espécie foram enviados ao Dr. Carlos Roberto Souza e Silva da Universidade de São Carlos (SP), para identificação.

b) Armadilhas tipo Moericke. Utilizaram-se 10 armadilhas com base no modelo originalmente descrito por MOERICKe (1951), mas com algumas modificações. As armadilhas consistiram de uma bandeja plástica, redonda, de $23 \mathrm{~cm}$ de diâmetro e $7 \mathrm{~cm}$ de altura, de coloração interna amarelo-ouro.

Nas laterais, dois orifícios de $1 \mathrm{~cm}$ de diâmetro dispostos em lados opostos, vedados com tela de nylon, evitaram o transbordamento do conteúdo e, conseqüentemente, a perda dos afídeos.

As armadilhas foram dispostas aleatoriamente na área, sendo semanalmente niveladas de acordo com a altura das plantas. O volume de líquido de cada bandeja foi substituído a cada coleta, com água e adicionado algumas gotas de detergente com o objetivo de quebrar a tensão superficial da água. 
As coletas foram realizadas a cada dois dias, com o auxílio de uma peneira de malha fina. Os insetos capturados foram transferidos para frascos de vidro contendo solução de álcool 70\% e, posteriormente, separados e contados, como descrito anteriormente.

\section{Resultados e Discussão}

Amostragens diretas nas plantas

Verificou-se que 14 dias após a germinação das plantas, iniciou-se um rápido crescimento da população de afídeos, tendo ocorrido um pico populacional aos 26 dias após a germinação, onde as ninfas representaram em média 36,65 indivíduos por planta e os adultos ápteros uma média de 13,10 indivíduos por planta. Todos os adultos ápteros observados nessas plantas foram identificados como A. gossypii.

Os alados estiveram presentes em pequena quantidade em todo o período de avaliação das plantas, tendo ocorrido em maior número 18 dias após a germinação com média de 0,95 indivíduo por planta. No entanto, esses indivíduos são importantes, pois são os responsáveis pela introdução de vírus fitopatogêncios na cultura (CAUQUIL, 1977).

Entre as formas aladas observadas nas plantas, registrou-se a ocorrência de três espécies: $A$. gossypii $(89,5 \%)$, Aphis spiraecola Patch, $(9,2 \%)$ e Rhophalosiphum padi (L.) (1,3\%). A relação entre a população de adultos alados e de adultos ápteros de $A$. gossypii foi comprovada pela análise de correlação $(\mathrm{r}=0,4543 ; \mathrm{p}<0,05 ;$ à medida que aumenta $\mathrm{a}$ população de adultos alados, aumenta a população de adultos ápteros, durante o período estudado.

A espécie A. spiraecola tem como plantas hospedeiras preferenciais rosáceas cultivadas ou silvestres, citros e espécies arbustivas da família Compositae (PeÑA-MartineZ, 1992). Segundo a mesma autora, esse afídeo também pode ser encontrado em solanáceas e cucurbitáceas como melão e abóbora, onde forma colônias com A. gossypii.

Os poucos indivíduos capturados dessa espécie foram observados no período intermediário das observações nas plantas (Figura 1).

A espécie R. padi, também considerada cosmopolita, têm como hospedeiros secundários as gramíneas (milho, cevada, aveia e trigo) e como hospedeiro primário Prunus sp. (Peña-Martinez, 1992). Apenas dois indivíduos dessa espécie foram encontrados nas plantas, provavelmente, de ocorrência acidental na cultura.

\section{Amostragens com as armadilhas}

Nas armadilhas tipo Moericke, foram capturadas 13 espécies de afídeos: $A$. gossypii, $A$. spiraecola, Geopemphigus floccosus (Moreira), Lizerius melanocallis (Quednau), Myzus persicae (Sulzer), Pentalonia nigronervosa Coquerel, Rhopalosiphum maidis (Fitch), R. padi, R. rufiabdominalis (Sasaki), Sipha flava (Forbes), Tetraneura nigriabdominalis (Sasak) ssp. bispina Hille Ris Lambers, Toxoptera citricidus (Kirkaldy) e Uroleucon ambrosiae (Thomas) forma lizerianum (E. E. Blanchard) (Quadro 1).

Quadro 1. Espécies capturadas em armadilhas tipo Moericke, no início do desenvolvimento das plantas de algodoeiro, cultivar CNPA ITA 90. Campo Verde (MT), 2001

\begin{tabular}{lcc}
\hline Espécies & Número total & Porcentagem \\
\hline Rhopalosiphum padi & 1.199 & 52,59 \\
Aphis spiraecola & 601 & 26,36 \\
Aphis gossypii & 202 & 8,86 \\
Rhopalosiphum maidis & 120 & 5,26 \\
Geopemphigus floccosus & 70 & 3,07 \\
Uroleucon ambrosiae & 35 & 1,54 \\
Rhopalosiphum rufiabdominalis & 30 & 1,32 \\
Myzus persicae & 8 & 0,35 \\
Sipha flava & 6 & 0,26 \\
Pentalonia nigronervosa & 3 & 0,13 \\
Tetraneura nigriabdominalis & 3 & 0,13 \\
Lizerius melanocallis & 2 & 0,09 \\
Toxoptera citricidus & 1 & 0,04 \\
\hline Total & 2.280 & 100,00 \\
\hline
\end{tabular}


O número de indivíduos alados presentes nas armadilhas totalizou 2.280 indivíduos nas 25 amostragens. A espécie que apresentou o maior número de exemplares foi $R$. padi (1.199 indivíduos), correspondendo a $52,59 \%$ do total amostrado, seguida de A. spiraecola (601) $26,36 \%$, A. gossypii (202) $8,86 \%$ e $R$. maidis (120) $5,26 \%$. As demais espécies variaram de 1 a 70 exemplares (Quadro 1). Duviard e MerCADIER (1973), utilizando o mesmo tipo de armadilha em algodoeiro na África, coletaram principalmente A. spiraecola $(34,2 \%)$, A. gossypii $(25,2 \%)$ e Schoudetenia bougainvilleae Theobald, $(22,4 \%)$.
A espécie R. padi foi capturada em maior número durante a primeira metade do período, ou seja, no primeiro mês após a germinação das plantas de algodoeiro (Figura 1). Resultados semelhantes foram observados para $R$. maidis, provavelmente em vista da presença de plantas de milheto próximos à área experimental. As espécies $A$. gossypii e $A$. spiraecola foram capturadas em todo o período amostral, entretanto, com os maiores picos populacionais ocorrendo na primeira metade do levantamento (30 dias iniciais) para $A$. gossypii e na segunda metade para $A$. spiraecola (Figura 1).
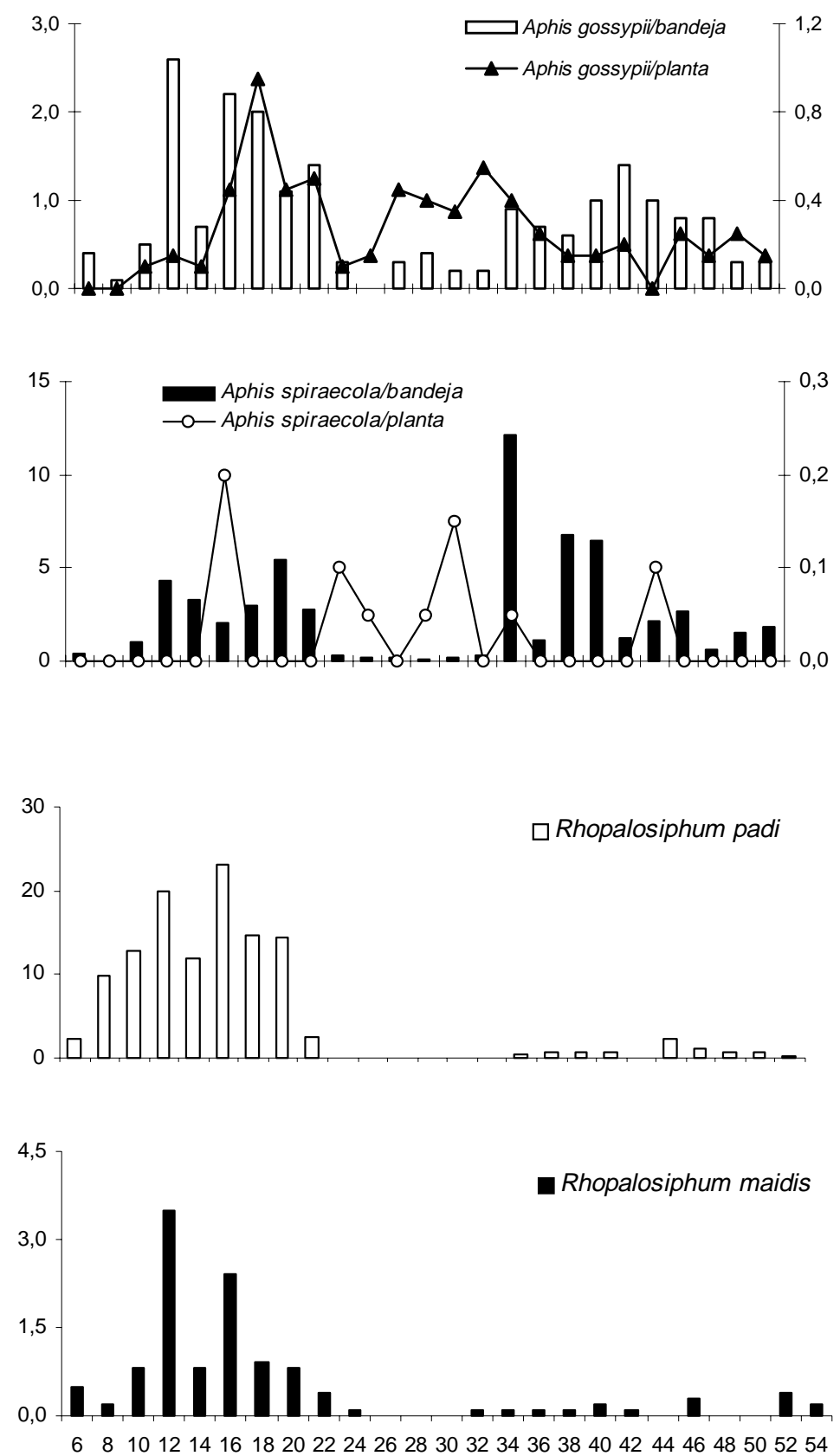

Figura 1. Quantidade de afídeos alados capturados em armadilhas tipo Moericke e visualizados em plantas de algodoeiro no início do desenvolvimento, cultivar CNPA ITA 90. Campo Verde (MT), 2001. 
Poucos indivíduos de $M$. persicae foram capturados (Quadro 1), apesar de citado como praga na cultura do algodoeiro no Brasil.

A população de alados de $A$. gossypii capturada nas bandejas apresentou uma correlação negativa $(\mathrm{r}=-0,4050 ; \mathrm{p}<0,05)$ com a população de adultos ápteros observados nas plantas. Para a população de alados da mesma espécie nas plantas e população de alados capturados nas bandejas não se observou correlação.

\section{Conclusões}

1. A. gossypii é a principal espécie observada diretamente nas plantas de algodoeiro, em Campo Verde (MT).

2. Nas armadilhas tipo Moericke, as principais espécies capturadas foram $R$. padi, A. spiraecola, $A$. gossypii e R. maidis.

3. Observou-se correlação positiva entre a população de alados e de ápteros de $A$. gossypii presentes nas plantas de algodoeiro.

\section{Referências Bibliográficas}

BLACKMAN, R. L.; EASTOP, V. P. Aphids on the world's crops: an identification guide. New York: John Wiley and Sons, 1984. 466p.

CALCAGNOLO, G.; SAUER, H. F. G. A influência do ataque dos pulgões na produção do algodão (Aphis gossypii, Glover 1876, Hom. Aphididae). Arquivos do Instituto Biológico, São Paulo, v.21, p.85-89, 1954.

CAUQUIL, J. Estudes ser une maladie d'origine virale du cotonnier: la maladie bleue. Coton et Fibres Tropicales, Paris, v.32, p.259-278, 1977.

CIA, E. Ocorrência e conhecimento das doenças de algodoeiro anual Gossypium hirsutum L. no Brasil. Summa Phytopathologica, Piracicaba, v.3, p.167-193, 1977.
COSTA, A. S.; JULIATTI, F. C.; RUANO, O. Algodão (Gossypium hirsutum L.): Doenças causadas por vírus. In: VALE, F.X.R.; ZAMBOLIM, L. (Eds.). Controle de doenças de plantas: grandes culturas. Viçosa, 1997. v.2, p.571-582.

DUVIARD, D; MERCADIER, G. Les invasions saisonnières de pucerons en culture cottonnière: origene et mécanismes. Coton et Fibres Tropicales, Paris, v.28, n.4, p.483-491, 1973.

FREIRE, E.C. Doença azul tem solução. Cultivar, Pelotas, n.1, p.64-65, 1999.

GODFREY, L.D.; ROSENHEIM, J.A.; GOODELL, P.B. Cotton aphid emerges as major pest in SJV cotton. California Agriculture, Oakland, v.54, n.6, p.26-29, 2000.

MOERICKE, V. Eine farbefalle zur kontrolle des fluges von blattläusen, inbesonder der pfirsichblattlaus Myzus persicae (Sulz.). Nachrichtenblatt für den Deutschen Pflanzenschutzdienstes, Stuttgart, v.3, p.23-24, 1951.

OCHOA, L. P. Lista de insectos dañinos y otras plagas en Colombia. 4.Ed. Bogotá: Instituto Colombiano Agropecuario, 1989. p.38. (Boletin Técnico, 43).

PEÑA-MARTINEZ, R. Identificacion de afidos de importancia agricola. In: URIAS-M, C.; RODRÍGUEZ-M, R.; ALEJANDREA, T. Afidos como vectores de virus en México. México: Centro de Fitopatologia, Montecillo, 1992. v.2, 135p.

SANTOS, W. J. Monitoramento e controle das pragas do algodoeiro. In: CIA, E.; FREIRE, E. C.; SANTOS, W. J. (Ed.). Cultura do algodoeiro, Piracicaba: Potafós, 1999, p.133-179.

STOETEZEL, M B.; MILLER, G. L.; O’BRIEN, P. J.; GRAVES, J. B. Aphids (Homoptera: Aphididae) colonizing cotton in the United States. Florida Entomologist, Winter Haven, v.79, n.2, p.193-205, 1996.

VENDRAMIM, J.D.; NAKANO, O. Aspectos biológicos de Aphis gossypii Glover, 1877 (Homoptera: Aphididae) em algodoeiro. Anais da Sociedade Entomológica do Brasil, Jaboticabal, v.10, n.2, p.163-173, 1981. 